\title{
Public Health Policy Perspective
}

\section{An Updated Assessment of Utilization of Interventional Pain Management Techniques in the Medicare Population: 2000 - 2013}

Laxmaiah Manchikanti, MD ${ }^{1,2}$, Vidyasagar Pampati, MSc1 ${ }^{1}$ Frank J.E. Falco, MD², and Joshua A. Hirsch, MD

From: ${ }^{1}$ Pain Management Center of Paducah, Paducah, $\mathrm{KY}$; and ${ }^{2}$ University of Louisville, Louisville, KY

3Mid Atlantic Spine \& Pain

Physicians, Newark, DE, and Temple University

Hospital, Philadelphia, PA; and ${ }^{4}$ Massachusetts Genera Hospital and Harvard Medical School, Boston, MA

Additional Author Affiliation information on P. E124

Address Correspondence: Laxmaiah Manchikanti, MD 2831 Lone Oak Road Paducah, KY 42003 E-mail: drlm@thepainmd.com

Disclaimer: There was no external funding in the preparation of this

Conflict of Interest: Dr. Manchikanti has provided limited consulting services to Semnur Pharmaceuticals, Incorporated, which is

developing nonparticulate steroids. Dr. Falco is a consultant for St. Jude Medical Inc. and Joimax Inc. Dr. Hirsch is a consultant for Medtronic.

Manuscript received: 03-03-2015 Accepted for publication: 03-09-20155

Free full manuscript: www.painphysicianjournal.

com
Background: The rapid increase in the prevalence of chronic pain and disability, and the explosion of interventional pain management associated health care costs are a major concern for our community. Further, the increasing utilization of numerous modalities of treatments in managing chronic pain, continue to escalate at a pace which may not be sustainable. There are multiple regulations in place to control the growth of health care expenditures which seem to have been largely ineffective. Among the various modalities utilized in managing chronic pain, interventional techniques have shown a significant increase in their utilization in the face of continued debate with respect to the accuracy of diagnostic interventions and the efficacy of therapeutic interventions.

Objective: To update and assess the utilization of interventional techniques in chronic pain management in fee-for-service Medicare population.

Study Design: An updated analysis of the growth of interventional techniques in managing chronic pain in fee-for-service Medicare beneficiaries from 2000 through 2013

Methods: The data were derived and analyzed utilizing the Centers for Medicare and Medicaid Services (CMS) Physician Supplier Procedure Summary Master Data from 2000 through 2013.

Results: From 2000 through 2013, in fee-for-service Medicare beneficiaries, the overall utilization of interventional techniques services increased $236 \%$ at an annual average growth of $9.8 \%$, whereas the per 100,000 Medicare population utilization increased $156 \%$ with an annual average growth of $7.5 \%$. During this period, the US population increased $12 \%$ with an annual average increase of $0.9 \%$, whereas those above 65 years of age increased $27 \%$ with an annual average increase of $1.9 \%$. Total Medicare beneficiaries increased $31 \%$ with an annual average increase of $2.1 \%$, with an overall increase of $64 \%$ for those above 65 years of age, an increase of $26 \%$, constituting $17 \%$ of the US population in 2013.

The overall increases in epidural and adhesiolysis procedures were $165 \%$ compared to $102 \%$ per 100,000 fee-for-service population with annual average increases of $7.8 \%$ and $5.6 \%$. Facet joint and sacroiliac joint injections increased $417 \%$ for services with an annual average increase of $13.5 \%$, whereas the rate per 100,000 fee-for-service Medicare beneficiaries increased 295\% with an annual average increase of $11.1 \%$.

Limitations: Limitations of this assessment include the lack of inclusion of participants from Medicare Advantage plans, lack of appropriate available data for state-wide utilization, and potential errors in documentation, coding, and billing.

Conclusion: This update once again shows a significant increase in interventional techniques in feefor-service Medicare beneficiaries from 2000 through 2013 with an increase of 156\% per 100,000 Medicare population with an annual average increase of $7.5 \%$. During this period the Medicare population increased $31 \%$ with an annual average increase of $2.1 \%$.

Key words: Chronic pain, chronic spinal pain, interventional pain management, interventional techniques, epidural injections, facet joint interventions, sacroiliac joint injections

Pain Physician 2015; 18:E115-E127 
T he increasing prevalence in chronic pain and disability, and the economic impact with increases in health care costs continue to be subjects of concern in the United States and across the globe (1-4). Specifically, spinal pain is highly pervasive and has been shown to contribute to disability, with 3 of 5 disorders constituting the cause of most years lived with disability in 2010 in the United States as well as worldwide which includes low back pain, other musculoskeletal disorders, and neck pain (1-10). Thus, not only costs, utilization, and appropriateness, but also complications related to various interventions in managing chronic pain, specifically spinal pain, have been debated extensively (1-4,11-34). Consequently, based on available reports, deaths due to acetaminophen were approximately 1,000 per year; nonsteroidal anti-inflammatory drug deaths, based on 1990 data, were 17,000; opioid deaths in 2012 were 16,235 , with deaths secondary to methadone alone of 4,400 in 2011. Deaths have escalated due to heroin and extensive liberalization of marijuana use. Surgical interventions, which have increased $137 \%$ for low back pain from 1998 to 2008, resulted in 1,012 deaths in $2008(15,20,22,27-32)$. Most of the focus has been on complications of interventional techniques and opioid use with increasing utilization (16-19,23-26,34). The Food and Drug Administration (FDA) has reported 131 deaths, of which 41 were secondary to arachnoiditis (2326). In addition, there was an unprecedented outbreak of fungal infection due to preservative-free, injectable methylprednisolone acetate in 2012 (33), affecting 76 facilities in 23 states and a total of 751 patients.

Published reports show that utilization of interventional techniques in managing chronic pain has been increasing substantially over the years. Manchikanti et al $(16,19)$, in an assessment of the population in the fee-for-service sector of Medicare, showed an overall increase of $228 \%$ and $177 \%$ per 100,000 Medicare beneficiaries from 2000 to 2011. In addition, they (17) also reported utilization and costs from 2000 to 2008. They found a $240 \%$ increase in costs and a $229 \%$ increase in procedures. They estimated the costs of spinal interventional techniques to be over $\$ 362$ million in 2000 , increasing to over $\$ 1.2$ billion in 2008 . Overall, per patient expenditures increased $19 \%$ and per visit expenditures increased $6 \%$ (17). Manchikanti et al (16), in describing accountable interventional pain management, a collaboration among practitioners, patients, payers, and government, discussed various issues related to escalating utilization, costs, and measures to reduce utilization and costs without affecting access to care. There have been multiple investigations from the Office of Inspector General in reference to the utilization of facet joint injections and transforaminal epidural injections $(35,36)$.

An emerging specialty, interventional pain management (IPM) and its techniques have their own definitions $(37,38)$. IPM is represented on Carrier Advisory Committees (39) in the United States. The specialty has a specific responsibility to provide medically necessary services while at the same time improving quality and curbing costs $(12,16,40)$; however, it is extremely difficult because of counter-acting forces with ever-changing coverage policies, regulations, an increasing population that has pain and disability, and finally health care reform and excessive utilization (1619,40-43). In addition, there also has been extensive debate on IPM's efficacy and effectiveness, including medical necessity, indications, and appropriateness of interventional techniques for managing chronic pain (44-53) with a case being made for and against these techniques by appropriately performed systematic reviews $(12,44-48)$, and others with inappropriate evidence synthesis and lack of appropriate methodology (39,50-52).

With the institution of national health care systems across the globe and affordable health care in the United States and an increasing aged population and Medicaid expansion in the United States secondary to the Affordable Care Act, interventional techniques have become the focus of attention in the United States. Further, Medicare has become a standard due to the organization being larger than any other insurance provider. Medicare continues to expand rapidly and all other payers, specifically Medicaid with its explosive expansion, seem to base their decisions on the policies of Medicare. As expected, multiple measures are applied by insurers and various governmental agencies across the globe to get a handle on exploding health care costs, specifically costs of chronic pain management with a focus on interventional techniques. However, the basic understanding of chronic pain itself and the proper and safe application of interventional techniques compared to various other clinical modalities available for managing chronic pain seems to be misunderstood. Further, utilization patterns, costs, and policies continue to emerge.

This study was undertaken to update previous assessments (19) about the utilization of interventional techniques in chronic pain from 2000 through 2013. 


\section{Methods}

The study was performed utilizing the Centers for Medicare and Medicaid Services (CMS) Physician Supplier Procedure Summary Master Data from 2000 through 2013 (54). The data were purchased from CMS by the American Society of Interventional Pain Physicians. This study was conducted with the internal resources of the primary author's practice without any external funding, either from industry or elsewhere. CMS's $100 \%$ data set is therefore expected to be unbiased and unpredictable in terms of any patient characteristics. Even though previous studies $(55,56)$ used only patients aged 65 or older, in this study we have used all patients enrolled in fee-for-service Medicare. A significant proportion of patients below the age of 65 receive interventional pain management services (17). Medicare represents the single largest health care payer in the United States, with over 51.9 million beneficiaries in 2013 (57). Thus, the procedures performed on Medicare beneficiaries represent a large proportion of the procedures for chronic pain being performed in the United States. Rates were calculated based on Medicare beneficiaries for the corresponding year and are reported as procedures per 100,000 Medicare beneficiaries.

For analysis, the Current Procedural Terminology procedure codes for interventional techniques [Epidural and Adhesiolysis procedures (62310, 62311, 64479, 64480, 64483, 64484, 62280, 62281, 62282, 62263, 62264); Facet Joint interventions and SI joint blocks (64470, 64472, 64475, 64476, 64490, 64491-new, 64492new, 64493-new, 64494-new, 64495-new, 64622, 64623, 64626, 64627, 64633-new, 64634-new, 64635-new, 64636-new, 27096); Discography and Disc decompression $(62290,62291,62287)$ other type of nerve blocks (64400, 64402, 64405, 64408, 64410, 64412, 64413, 64417, 64420, 64421, 64425, 64430, 64445, 64505, $64510,64520,64530,64600,64605,64610,64613,64620$, $64630,64640,64680)$ ] were identified for 2000 through 2013. The data were then tabulated based on the place of service - facility (ambulatory surgery center, hospital outpatient department) or nonfacility (office). The calculated data included the number of interventional pain management services and the rate of services per 100,000 Medicare beneficiaries.

Various specialties were described as providers: interventional pain management -09 , pain medicine -72 , anesthesiology -05 , physical medicine and rehabilitation -25 , neurology -13 , psychiatry -26 , all constituting interventional pain management; orthopedic surgery -20 , general surgery-17 and neurosurgery -14 as a surgical group; radiology specialties as a separate group (-30 diagnostic radiology, -94 interventional radiology); all other physicians as another group; and all other providers were considered as other providers.

\section{Statistical Analysis}

The data were analyzed using SPSS 9.0 statistical software (SPSS, Inc., Chicago, IL) Microsoft Access 2003, and Microsoft Excel 2003 (Microsoft, Redmond, WA). The procedure rates were calculated per 100,000 Medicare beneficiaries.

\section{Results}

\section{Population Characteristics}

As illustrated in Table 1, the number of Medicare beneficiaries increased from 39.632 million in 2000 to 51.900 million in 2013, an increase of $31 \%$ compared to an increase of $12 \%$ in the US population.

\section{Utilization Characteristics}

Table 2 illustrates a summary of the frequency of utilization in various categories of interventional techniques in Medicare beneficiaries from 2000 to 2013.

Overall, the increase in interventional pain management procedures from 2000 to 2013 was 236\%, with a $156 \%$ increase per 100,000 Medicare beneficiaries. The increases were highest for facet joint interventions and sacroiliac joint blocks, with $417 \%$ total and $295 \%$ per 100,000 Medicare beneficiaries, followed by $165 \%$ and $102 \%$ for epidural and adhesiolysis procedures, $161 \%$ and $99 \%$ for other types of nerve blocks and finally, a $3 \%$ increase and $22 \%$ decrease for disc procedures. The geometric average of annual increases was $9.8 \%$ overall with $13.5 \%$ for facet joint interventions and sacroiliac joint blocks and $7.8 \%$ for epidural and adhesiolysis procedures.

Fig. 1 illustrates the distribution of procedural characteristics from 2000 to 2013.

\section{Specialty Characteristics}

Tables 3 and 4 illustrate procedural characteristics based on specialty. Overall increases were $236 \%$ with a $156 \%$ increase per 100,000 Medicare beneficiaries. For interventional pain management, these increases were $268 \%$ and $181 \%$; for surgical specialties, including neurosurgery, orthopedic surgery and general surgery, increases were $101 \%$ and $54 \%$; for radiology, they were $194 \%$ and $125 \%$; for other physicians, they were $60 \%$ and $22 \%$; and for other providers, they were $323 \%$ and 
Pain Physician: March/April 2015; 18:E115-E127

Table 1. Characteristics of Medicare beneficiaries and utilization of interventional pain management services.

\begin{tabular}{|c|c|c|c|c|c|c|c|c|c|c|}
\hline \multirow[b]{2}{*}{ Year } & \multicolumn{3}{|c|}{ U.S. Population } & \multicolumn{4}{|c|}{ Medicare Beneficiaries } & \multicolumn{3}{|c|}{ IPM Services } \\
\hline & $\begin{array}{c}\text { Population } \\
\quad(, 000)\end{array}$ & $\begin{array}{c}\geq 65 \\
\text { Years } \\
(, 000)\end{array}$ & Percent & Medicare & $\begin{array}{l}\% \text { to } \\
\text { U.S. }\end{array}$ & $\begin{array}{c}\geq 65 \text { years } \\
(, 000) \\
\text { (Percent) }\end{array}$ & $\begin{array}{c}<65 \text { years } \\
(, 000) \\
\text { Percent }\end{array}$ & Services & $\begin{array}{c}\% \text { of } \\
\text { Change from } \\
\text { Previous } \\
\text { Year }\end{array}$ & $\begin{array}{c}\text { Rate } \\
\text { Per } \\
\mathbf{1 0 0 , 0 0 0}\end{array}$ \\
\hline Y2000 & 282,172 & 35,077 & $12.40 \%$ & 39,632 & $14.0 \%$ & $\begin{array}{c}34,262 \\
(86.5 \%)\end{array}$ & $\begin{array}{c}5,370 \\
(13.5 \%)\end{array}$ & $1,469,495$ & - & 3,708 \\
\hline Y2001 & 285,040 & 35,332 & $12.40 \%$ & 40,045 & $14.0 \%$ & $\begin{array}{c}34,478 \\
(86.1 \%)\end{array}$ & $\begin{array}{c}5,567 \\
(13.9 \%)\end{array}$ & $1,760,456$ & $19.8 \%$ & 4,396 \\
\hline Y2002 & 288,369 & 35,605 & $12.30 \%$ & 40,503 & $14.0 \%$ & $\begin{array}{c}34,698 \\
(85.7 \%) \\
\end{array}$ & $\begin{array}{c}5,805 \\
(14.3 \%) \\
\end{array}$ & $2,183,052$ & $24.0 \%$ & 5,390 \\
\hline Y2003 & 290,211 & 35,952 & $12.40 \%$ & 41,126 & $14.2 \%$ & $\begin{array}{c}35,050 \\
(85.2 \%) \\
\end{array}$ & $\begin{array}{c}6,078 \\
(14.8 \%) \\
\end{array}$ & $2,559,323$ & $17.2 \%$ & 6,223 \\
\hline Y2004 & 292,892 & 36,302 & $12.40 \%$ & 41,729 & $14.2 \%$ & $\begin{array}{c}35,328 \\
(84.7 \%)\end{array}$ & $\begin{array}{c}6,402 \\
(15.3 \%)\end{array}$ & $3,335,047$ & $30.3 \%$ & 7,992 \\
\hline Y2005 & 295,561 & 36,752 & $12.40 \%$ & 42,496 & $14.4 \%$ & $\begin{array}{c}35,777 \\
(84.2 \%)\end{array}$ & $\begin{array}{c}6,723 \\
(15.8 \%)\end{array}$ & $3,660,699$ & $9.8 \%$ & 8,614 \\
\hline Y2006 & 299,395 & 37,264 & $12.40 \%$ & 43,339 & $14.5 \%$ & $\begin{array}{c}36,317 \\
(83.8 \%) \\
\end{array}$ & $\begin{array}{c}7,022 \\
(16.2 \%) \\
\end{array}$ & $4,146,124$ & $13.3 \%$ & 9,567 \\
\hline Y2007 & 301,290 & 37,942 & $12.60 \%$ & 44,263 & $14.7 \%$ & $\begin{array}{c}36,966 \\
(83.5 \%)\end{array}$ & $\begin{array}{c}7,297 \\
(16.5 \%)\end{array}$ & $4,111,127$ & $-0.8 \%$ & 9,288 \\
\hline Y2008 & 304,056 & 38,870 & $12.80 \%$ & 45,412 & $14.9 \%$ & $\begin{array}{c}37,896 \\
(83.4 \%)\end{array}$ & $\begin{array}{c}7,516 \\
(16.6 \%)\end{array}$ & $4,433,411$ & $7.8 \%$ & 9,763 \\
\hline Y2009 & 307,006 & 39,570 & $12.90 \%$ & 45,801 & $14.9 \%$ & $\begin{array}{c}38,177 \\
(83.4 \%)\end{array}$ & $\begin{array}{c}7,624 \\
(16.6 \%)\end{array}$ & $4,645,679$ & $4.8 \%$ & 10,143 \\
\hline Y2010 & 308,746 & 40,268 & $13.00 \%$ & 46,914 & $15.2 \%$ & $\begin{array}{c}38,991 \\
(83.1 \%)\end{array}$ & $\begin{array}{c}7,923 \\
(16.9 \%)\end{array}$ & $4,578,977$ & $-1.4 \%$ & 9,760 \\
\hline Y2011 & 311,583 & 41,370 & $13.28 \%$ & 48,300 & $15.5 \%$ & $\begin{array}{c}40,000 \\
(82.8 \%) \\
\end{array}$ & $\begin{array}{c}8,300 \\
(17.2 \%) \\
\end{array}$ & $4,815,673$ & $5.2 \%$ & 9,970 \\
\hline Y2012 & 313,874 & 43,144 & $13.75 \%$ & 50,300 & $16.0 \%$ & $\begin{array}{c}41,900 \\
(83.3 \%) \\
\end{array}$ & $\begin{array}{c}8,500 \\
(16.9 \%)\end{array}$ & $4,947,974$ & $2.7 \%$ & 9,837 \\
\hline Y2013 & 316,129 & 44,704 & $14.14 \%$ & 51,900 & $16.4 \%$ & $\begin{array}{c}43,100 \\
(83.0 \%) \\
\end{array}$ & $\begin{array}{c}8,800 \\
(17.0 \%) \\
\end{array}$ & $4,932,950$ & $-0.3 \%$ & 9,505 \\
\hline Change & $12.0 \%$ & $27.4 \%$ & - & $31.0 \%$ & - & $25.8 \%$ & $63.9 \%$ & $236 \%$ & - & $156 \%$ \\
\hline GM & $0.9 \%$ & $1.9 \%$ & - & $2.1 \%$ & - & $1.8 \%$ & $3.9 \%$ & $9.8 \%$ & - & $7.5 \%$ \\
\hline
\end{tabular}

*(Excluding continuous epidurals, intraarticular injections, trigger point and ligament injections, peripheral nerve blocks, vertebral augmentation procedures, and implantables)

$223 \%$ increase overall and per 100,000 Medicare beneficiaries. Fig. 2 illustrates the distribution of specialty characteristics.

\section{Discussion}

Interventional techniques for chronic pain have increased dramatically from 2000 to 2013. The increases were present in all settings and by all types of specialists. Over this period from 2000 to 2013, beneficiaries increased $31 \%$, whereas overall interventional pain management services increased $236 \%$, whereas rate per 100,000 Medicare beneficiaries increased 156\%. The study also showed an exponential increase in facet joint interventions with a rate of $295 \%$ increase per 100,000 beneficiaries and annual average growth of $11.1 \%$, more than any other modality. Overall, average annual increases were $7.5 \%$ per 100,000 Medicare beneficiaries.

The results of this evaluation of growth patterns are similar to previous evaluations $(17,19,55,56,58-$ $60)$ although they differ in select aspects. Friedly et 
Assessment of Utilization of IPM Techniques in the Medicare Population

Table 2. Updated utilization of frequency of interventional techniques in the Medicare population from 2000 to 2013.

\begin{tabular}{|c|c|c|c|c|c|c|c|c|c|c|c|}
\hline \multirow{2}{*}{ Year } & \multicolumn{2}{|c|}{$\begin{array}{l}\text { Epidural and } \\
\text { adhesiolysis } \\
\text { procedures }\end{array}$} & \multicolumn{2}{|c|}{$\begin{array}{c}\text { Facet joint } \\
\text { interventions } \\
\text { and SI joint blocks }\end{array}$} & \multicolumn{2}{|c|}{$\begin{array}{c}\text { Disc Procedures } \\
\text { (discography } \\
\text { \& disc } \\
\text { decompression) }\end{array}$} & \multicolumn{2}{|c|}{$\begin{array}{c}\text { Other types of } \\
\text { nerve blocks }\end{array}$} & \multicolumn{3}{|c|}{ Total* } \\
\hline & $\begin{array}{c}\text { Services } \\
\text { (Facility \%) }\end{array}$ & Rate & $\begin{array}{c}\text { Services } \\
\text { (Facility } \\
\% \text { ) }\end{array}$ & Rate & $\begin{array}{c}\text { Services } \\
\text { (Facility } \\
\% \text { ) }\end{array}$ & Rate & $\begin{array}{c}\text { Services } \\
\text { (Facility\%) }\end{array}$ & Rate & $\begin{array}{c}\text { Services } \\
\text { (Facility\%) }\end{array}$ & $\begin{array}{l}\text { Change } \\
\text { from } \\
\text { previous } \\
\text { year }\end{array}$ & Rate \\
\hline 2000 & $\begin{array}{c}860,787 \\
(79 \%)\end{array}$ & 2,172 & $\begin{array}{c}424,796 \\
(67 \%)\end{array}$ & 1,072 & $\begin{array}{l}14,983 \\
(87 \%)\end{array}$ & 38 & $\begin{array}{c}168,929 \\
(42 \%)\end{array}$ & 426 & $\begin{array}{c}1,469,495 \\
(72 \%)\end{array}$ & - & 3,708 \\
\hline 2001 & $\begin{array}{c}1,013,552 \\
(78 \%)\end{array}$ & 2,531 & $\begin{array}{c}543,509 \\
(62 \%)\end{array}$ & 1,357 & $\begin{array}{l}17,229 \\
(87 \%)\end{array}$ & 43 & $\begin{array}{c}186,166 \\
(38 \%)\end{array}$ & 465 & $\begin{array}{c}1,760,456 \\
(69 \%)\end{array}$ & $19.8 \%$ & 4,396 \\
\hline 2002 & $\begin{array}{c}1,199,324 \\
(74 \%)\end{array}$ & 2,961 & $\begin{array}{c}708,186 \\
(58 \%)\end{array}$ & 1,748 & $\begin{array}{l}20,194 \\
(81 \%)\end{array}$ & 50 & $\begin{array}{c}255,348 \\
(30 \%)\end{array}$ & 630 & $\begin{array}{c}2,183,052 \\
(64 \%)\end{array}$ & $24.0 \%$ & 5,390 \\
\hline 2003 & $\begin{array}{c}1,370,862 \\
(71 \%) \\
\end{array}$ & 3,333 & $\begin{array}{c}884,035 \\
(53 \%) \\
\end{array}$ & 2,150 & $\begin{array}{l}24,362 \\
(80 \%) \\
\end{array}$ & 59 & $\begin{array}{c}280,064 \\
(27 \%)\end{array}$ & 681 & $\begin{array}{c}2,559,323 \\
(60 \%) \\
\end{array}$ & $17.2 \%)$ & 6,223 \\
\hline 2004 & $\begin{array}{c}1,637,494 \\
(65 \%)\end{array}$ & 3,924 & $\begin{array}{c}1,354,242 \\
(46 \%)\end{array}$ & 3,245 & $\begin{array}{l}24,263 \\
(79 \%)\end{array}$ & 58 & $\begin{array}{c}319,048 \\
(26 \%)\end{array}$ & 765 & $\begin{array}{c}3,335,047 \\
(54 \%)\end{array}$ & $30.3 \%$ & 7,992 \\
\hline 2005 & $\begin{array}{c}1,776,153 \\
(65 \%)\end{array}$ & 4,180 & $\begin{array}{c}1,501,222 \\
(47 \%)\end{array}$ & 3,533 & $\begin{array}{l}27,950 \\
(78 \%)\end{array}$ & 66 & $\begin{array}{c}355,374 \\
(26 \%)\end{array}$ & 836 & $\begin{array}{c}3,660,699 \\
(54 \%)\end{array}$ & $9.8 \%$ & 8,614 \\
\hline 2006 & $\begin{array}{c}1,870,440 \\
(63 \%)\end{array}$ & 4,316 & $\begin{array}{c}1,896,688 \\
(40 \%)\end{array}$ & 4,376 & $\begin{array}{c}27,432 \\
(75 \%)\end{array}$ & 63 & $\begin{array}{c}351,564 \\
(26 \%)\end{array}$ & 811 & $\begin{array}{c}4,146,124 \\
(49 \%)\end{array}$ & $13.3 \%$ & 9,567 \\
\hline 2007 & $\begin{array}{c}1,940,454 \\
(62 \%)\end{array}$ & 4,384 & $\begin{array}{c}1,820,695 \\
(46 \%)\end{array}$ & 4,113 & $\begin{array}{l}25,688 \\
(73 \%) \\
\end{array}$ & 58 & $\begin{array}{c}324,290 \\
(30 \%)\end{array}$ & 733 & $\begin{array}{c}4,111,127 \\
(52 \%)\end{array}$ & $-0.8 \%$ & 9,288 \\
\hline 2008 & $\begin{array}{c}2,041,155 \\
(61 \%)\end{array}$ & 4,495 & $\begin{array}{c}1,974,999 \\
(46 \%)\end{array}$ & 4,349 & $\begin{array}{l}27,735 \\
(70 \%)\end{array}$ & 61 & $\begin{array}{c}389,522 \\
(29 \%)\end{array}$ & 858 & $\begin{array}{c}4,433,411 \\
(51 \%)\end{array}$ & $7.8 \%$ & 9,763 \\
\hline 2009 & $\begin{array}{c}2,136,035 \\
(59 \%)\end{array}$ & 4,664 & $\begin{array}{c}2,111,700 \\
(46 \%)\end{array}$ & 4,611 & $\begin{array}{l}25,929 \\
(69 \%)\end{array}$ & 57 & $\begin{array}{c}372,015 \\
(67 \%)\end{array}$ & 812 & $\begin{array}{c}4,645,679 \\
(49 \%)\end{array}$ & $4.8 \%$ & 10,143 \\
\hline 2010 & $\begin{array}{c}2,226,486 \\
(57 \%)\end{array}$ & 4,746 & $\begin{array}{c}1,937,582 \\
(48 \%)\end{array}$ & 4,130 & $\begin{array}{l}22,003 \\
(62 \%)\end{array}$ & 47 & $\begin{array}{c}392,906 \\
(34 \%)\end{array}$ & 838 & $\begin{array}{c}4,578,977 \\
(52 \%)\end{array}$ & $-1.4 \%$ & 9,760 \\
\hline 2011 & $\begin{array}{c}2,309,906 \\
(58 \%)\end{array}$ & 4,782 & $\begin{array}{c}2,064,227 \\
(50 \%)\end{array}$ & 4,274 & $\begin{array}{l}19,104 \\
(61 \%)\end{array}$ & 40 & $\begin{array}{c}422,436 \\
(66 \%)\end{array}$ & 875 & $\begin{array}{c}4,815,673 \\
(48 \%)\end{array}$ & $5.2 \%$ & 9,970 \\
\hline 2012 & $\begin{array}{c}2,324,563 \\
(58 \%)\end{array}$ & 4,621 & $\begin{array}{c}2,159,057 \\
(50 \%)\end{array}$ & 4,292 & $\begin{array}{l}18,017 \\
(57 \%)\end{array}$ & 36 & $\begin{array}{c}446,337 \\
(36 \%)\end{array}$ & 887 & $\begin{array}{c}4,947,974 \\
(53 \%)\end{array}$ & $2.7 \%$ & 9,837 \\
\hline 2013 & $\begin{array}{c}2,278,790 \\
(58 \%)\end{array}$ & 4,391 & $\begin{array}{c}2,197,766 \\
(51 \%)\end{array}$ & 4,235 & $\begin{array}{l}15,394 \\
(51 \%)\end{array}$ & 30 & $\begin{array}{c}441,000 \\
(37 \%)\end{array}$ & 850 & $\begin{array}{c}4,932,950 \\
(53 \%)\end{array}$ & $-0.3 \%$ & 9,505 \\
\hline Change & $165 \%$ & $102 \%$ & $417 \%$ & $295 \%$ & $3 \%$ & $-22 \%$ & $161 \%$ & $99 \%$ & $236 \%$ & - & $156 \%$ \\
\hline Average & $7.80 \%$ & $5.6 \%$ & $13.50 \%$ & $11.1 \%$ & $0.20 \%$ & $-1.8 \%$ & $7.70 \%$ & $5.4 \%$ & $9.80 \%$ & - & $7.5 \%$ \\
\hline
\end{tabular}

Rate per 100,000 Medicare Beneficiaries; IPM - Interventional Pain Management

Change: Change from 2000 to 2013; Average - Geometric average annual change

Epidural and Adhesiolysis procedures: 62310, 62311, 64479, 64480, 64483, 64484, 62280, 62281, 62282, 62263, 62264

Facet Joint interventions and SI joint blocks: 64470, 64472, 64475, 64476, 64490, 64491 (new), 64492 (new), 64493 (new), 64494 (new), 64495

(new), 64622, 64623, 64626, 64627, 64633 (new), 64634 (new), 64635 (new), 64636 (new), 27096

Discography and Disc decompression: 62290, 62291, 62287

Other type of nerve blocks: $64400,64402,64405,64408,64410,64412,64413,64417,64420,64421,64425,64430,64445,64505,64510,64520$,

$64530,64600,64605,64610,64613,64620,64630,64640,64680$

al $(55,56)$ focused on the escalating use of injection therapies coupled with a lack of evidence for managing chronic low back pain and geographic variation in epidural steroid injections, reaching inaccurate conclusions (61). These results no longer represent the present day atmosphere. Abbott et al (18) basically utilized an inappropriate concept and hypothesis.

Some critics of increasing utilization continue to claim interventional techniques lack evidence, and question if back pain is increasing $(15,62-64)$. How- 
Pain Physician: March/April 2015; 18:E115-E127

Table 3. Frequency of utilization of interventional pain management techniques from 2000 to 2013, in Medicare recipients.

\begin{tabular}{|c|c|c|c|c|c|c|c|c|c|c|c|c|}
\hline \multirow[t]{2}{*}{ Specialty } & \multicolumn{2}{|c|}{$\begin{array}{c}\text { Interventional } \\
\text { Pain } \\
\text { Management \# }\end{array}$} & \multicolumn{2}{|c|}{$\begin{array}{l}\text { Surgical (neuro, } \\
\text { general \& } \\
\text { orthopedic) }\end{array}$} & \multicolumn{2}{|c|}{$\begin{array}{c}\text { Radiology } \\
\text { (interventional } \\
\text { \& diagnostic) }\end{array}$} & \multicolumn{2}{|c|}{$\begin{array}{c}\text { Other } \\
\text { Physicians }\end{array}$} & \multicolumn{2}{|c|}{$\begin{array}{c}\text { Other Providers } \\
\text { (CRNA, NP \& } \\
\text { PA) }\end{array}$} & \multicolumn{2}{|c|}{ Total } \\
\hline & Services & Rate & Services & Rate & Services & Rate & Services & Rate & Services & Rate & Services* & Rate \\
\hline 2000 & $\begin{array}{c}1,176,541 \\
(80.1 \%)\end{array}$ & 2,969 & $\begin{array}{l}92,126 \\
(6.3 \%)\end{array}$ & 232 & $\begin{array}{l}40,491 \\
(2.8 \%)\end{array}$ & 102 & $\begin{array}{c}145,100 \\
(9.9 \%)\end{array}$ & 366 & $\begin{array}{l}15,237 \\
(1.0 \%)\end{array}$ & 38 & $\begin{array}{c}1,469,495 \\
(72 \%)\end{array}$ & 3,708 \\
\hline 2001 & $\begin{array}{c}1,389,569 \\
(78.9 \%) \\
\end{array}$ & 3,470 & $\begin{array}{c}105,075 \\
(6.0 \%) \\
\end{array}$ & 262 & $\begin{array}{l}48,978 \\
(2.8 \%) \\
\end{array}$ & 122 & $\begin{array}{l}196,311 \\
(11.2 \%) \\
\end{array}$ & 490 & $\begin{array}{l}20,524 \\
(1.2 \%) \\
\end{array}$ & 51 & $\begin{array}{c}1,760,456 \\
(69 \%)\end{array}$ & 4,396 \\
\hline 2002 & $\begin{array}{c}1,755,521 \\
(80.4 \%)\end{array}$ & 4,334 & $\begin{array}{c}123,403 \\
(5.7 \%)\end{array}$ & 305 & $\begin{array}{l}62,295 \\
(2.9 \%)\end{array}$ & 154 & $\begin{array}{l}218,870 \\
(10.0 \%)\end{array}$ & 540 & $\begin{array}{l}22,963 \\
(1.1 \%)\end{array}$ & 57 & $\begin{array}{c}2,183,052 \\
(64 \%)\end{array}$ & 5,390 \\
\hline 2003 & $\begin{array}{c}2,098,053 \\
(82.0 \%)\end{array}$ & 5,102 & $\begin{array}{c}133,165 \\
(5.2 \%)\end{array}$ & 324 & $\begin{array}{l}77,160 \\
(3.0 \%)\end{array}$ & 188 & $\begin{array}{c}229,010 \\
(8.9 \%)\end{array}$ & 557 & $\begin{array}{l}21,935 \\
(0.9 \%)\end{array}$ & 53 & $\begin{array}{c}2,559,323 \\
(60 \%)\end{array}$ & 6,223 \\
\hline 2004 & $\begin{array}{c}2,718,622 \\
(81.5 \%)\end{array}$ & 6,515 & $\begin{array}{c}168,669 \\
(5.1 \%)\end{array}$ & 404 & $\begin{array}{l}91,892 \\
(2.8 \%)\end{array}$ & 220 & $\begin{array}{c}329,705 \\
(9.9 \%)\end{array}$ & 790 & $\begin{array}{l}26,519 \\
(0.8 \%)\end{array}$ & 64 & $\begin{array}{c}3,335,047 \\
(54 \%)\end{array}$ & 7,992 \\
\hline 2005 & $\begin{array}{c}2,976,908 \\
(81.3 \%)\end{array}$ & 7,005 & $\begin{array}{c}183,972 \\
(5.0 \%)\end{array}$ & 433 & $\begin{array}{c}101,586 \\
(2.8 \%)\end{array}$ & 239 & $\begin{array}{l}367,303 \\
(10.0 \%)\end{array}$ & 864 & $\begin{array}{l}30,930 \\
(0.8 \%)\end{array}$ & 73 & $\begin{array}{c}3,660,699 \\
(54 \%)\end{array}$ & 8,614 \\
\hline 2006 & $\begin{array}{c}3,196,190 \\
(77.1 \%)\end{array}$ & 7,375 & $\begin{array}{c}211,580 \\
(5.1 \%)\end{array}$ & 488 & $\begin{array}{c}110,472 \\
(2.7 \%)\end{array}$ & 255 & $\begin{array}{l}589,835 \\
(14.2 \%)\end{array}$ & 1361 & $\begin{array}{l}38,047 \\
(0.9 \%)\end{array}$ & 88 & $\begin{array}{c}4,146,124 \\
(49 \%)\end{array}$ & 9,567 \\
\hline 2007 & $\begin{array}{c}3,405,892 \\
(82.8 \%)\end{array}$ & 7,695 & $\begin{array}{c}231,170 \\
(5.6 \%)\end{array}$ & 522 & $\begin{array}{c}111,423 \\
(2.7 \%)\end{array}$ & 252 & $\begin{array}{c}323,021 \\
(7.9 \%)\end{array}$ & 730 & $\begin{array}{l}39,621 \\
(1.0 \%)\end{array}$ & 90 & $\begin{array}{c}4,111,127 \\
(52 \%)\end{array}$ & 9,288 \\
\hline 2008 & $\begin{array}{c}3,670,828 \\
(82.8 \%)\end{array}$ & 8,083 & $\begin{array}{c}247,125 \\
(5.6 \%)\end{array}$ & 544 & $\begin{array}{c}117,388 \\
(2.6 \%)\end{array}$ & 258 & $\begin{array}{c}354,877 \\
(8.0 \%)\end{array}$ & 781 & $\begin{array}{l}43,193 \\
(1.0 \%)\end{array}$ & 95 & $\begin{array}{c}4,433,411 \\
(51 \%)\end{array}$ & 9,763 \\
\hline 2009 & $\begin{array}{c}3,879,520 \\
(83.5 \%)\end{array}$ & 8,470 & $\begin{array}{c}273,436 \\
(5.9 \%)\end{array}$ & 597 & $\begin{array}{c}123,228 \\
(2.7 \%)\end{array}$ & 269 & $\begin{array}{c}324,729 \\
(7.0 \%)\end{array}$ & 709 & $\begin{array}{l}44,766 \\
(1.0 \%)\end{array}$ & 98 & $\begin{array}{c}4,645,679 \\
(49 \%)\end{array}$ & 10,143 \\
\hline 2010 & $\begin{array}{c}3,917,426 \\
(85.6 \%)\end{array}$ & 8,350 & $\begin{array}{c}222,784 \\
(4.9 \%)\end{array}$ & 475 & $\begin{array}{c}121,127 \\
(2.6 \%)\end{array}$ & 258 & $\begin{array}{c}265,771 \\
(5.8 \%)\end{array}$ & 567 & $\begin{array}{l}51,869 \\
(1.1 \%)\end{array}$ & 111 & $\begin{array}{c}4,578,977 \\
(52 \%)\end{array}$ & 9,760 \\
\hline 2011 & $\begin{array}{c}4,159,585 \\
(86.4 \%)\end{array}$ & 8,612 & $\begin{array}{c}206,805 \\
(4.3 \%)\end{array}$ & 428 & $\begin{array}{c}127,614 \\
(2.6 \%)\end{array}$ & 264 & $\begin{array}{c}259,177 \\
(5.4 \%)\end{array}$ & 537 & $\begin{array}{l}62,492 \\
(1.3 \%)\end{array}$ & 129 & $\begin{array}{c}4,815,673 \\
(48 \%)\end{array}$ & 9,970 \\
\hline 2012 & $\begin{array}{c}4,302,121 \\
(86.9 \%)\end{array}$ & 8,553 & $\begin{array}{c}197,982 \\
(4.0 \%)\end{array}$ & 394 & $\begin{array}{c}129,823 \\
(2.6 \%)\end{array}$ & 258 & $\begin{array}{c}244,626 \\
(4.9 \%)\end{array}$ & 486 & $\begin{array}{l}73,422 \\
(1.5 \%)\end{array}$ & 146 & $\begin{array}{c}4,947,974 \\
(53 \%)\end{array}$ & 9,837 \\
\hline 2013 & $\begin{array}{c}4,331,789 \\
(87.8 \%)\end{array}$ & 8,346 & $\begin{array}{c}185,630 \\
(3.8 \%)\end{array}$ & 358 & $\begin{array}{c}119,172 \\
(2.4 \%)\end{array}$ & 230 & $\begin{array}{c}231,899 \\
(4.7 \%)\end{array}$ & 447 & $\begin{array}{l}64,460 \\
(1.3 \%)\end{array}$ & 124 & $\begin{array}{c}4,932,950 \\
(53 \%)\end{array}$ & 9,505 \\
\hline Change & $268 \%$ & $181 \%$ & $101 \%$ & $54 \%$ & $194 \%$ & $125 \%$ & $60 \%$ & $22 \%$ & $323 \%$ & $223 \%$ & $236 \%$ & $156 \%$ \\
\hline GM & $10.5 \%$ & $8.3 \%$ & $5.5 \%$ & $3.4 \%$ & $8.7 \%$ & $6.4 \%$ & $3.7 \%$ & $1.5 \%$ & $11.7 \%$ & $9.4 \%$ & $9.8 \%$ & $7.5 \%$ \\
\hline
\end{tabular}

Rate - IPM services per 100,000 Medicare Beneficiaries - GM - Geometric average annual change

( )percentage of row total

*(Excluding continuous epidurals, intraarticular injections, trigger point and ligament injections, peripheral nerve blocks, vertebral augmentation procedures, and implantables)

ever, disability secondary to spinal pain, health, and economic impact are increasing at an explosive rate, along with evidence of the increase in the prevalence of spinal pain (1-10,13,65-73). In fact, Freburger et al (5) showed an increase in low back pain in North Carolina of $162 \%$, from $3.9 \%$ to $10.2 \%$ over a period of 14 years from 1992 to 2006. Our understanding of the impact of chronic pain has changed over the years, specifically with its comorbid disorders and functional limitations. The impact of chronic pain has been described by various organizations as it fits their needs. The Institute of Medicine (1), based on data from Gaskin and Richard
(2), estimated chronic pain in 100 million patients to have a cost of $\$ 650$ billion; however, these estimations are inaccurate in that moderate and severe persistent pain contributed to 44.9 million persons, costing approximately $\$ 100$ billion a year in the United States (74). Further, the FDA commissioner also used these numbers to justify the approval of Zohydro ER (Zogenix Inc., San Diego, CA) which faced stiff opposition from multiple organizations, Congress, and governors (74).

There are several limitations to our study; for example the lack of inclusion of participants in Medicare Advantage plans and potential coding errors. In 


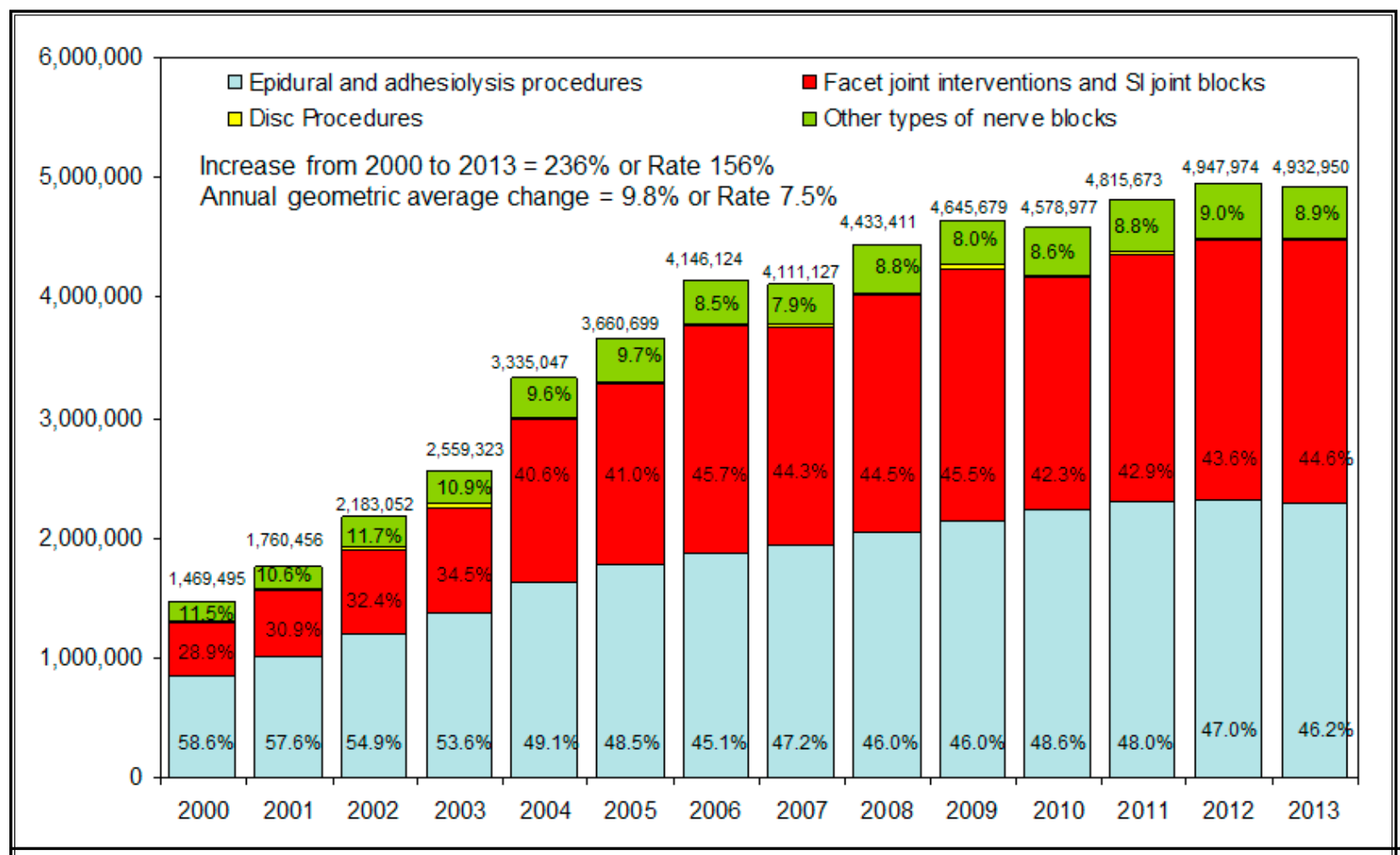

Fig. 1. Distribution of procedural characteristics by type of procedures from 2000 to 2013.

contrast to previous studies $(55,56)$, we employed all patients receiving Medicare either below the age of 65 or over the age of 65 . This inclusion is extremely important because patients below the age of 65 represent a significant proportion of patients receiving interventional techniques with higher frequency (4.50 vs. 3.35 services per patient) in 2006 (60). Further, by limiting to the Medicare database, this study has not evaluated other insurance providers including Medicaid, workmen's compensation and other carriers. However, the data from the FDA (26), shows utilization of epidural injections in Medicare and non-Medicare population. This data showed that over a period of approximately 5 years, 6.6 million epidural injections were administered to 1.4 million patients over the age of 65 years. Thus, even this data has missed those of less than 65 years of age and Medicare Advantage plans. The FDA data also showed among other payers, in those who were aged 0 to 59 years, with 150,572 patients receiving 262,301 epidural injections in 2012. Thus, the present data correlates with the data provided by the FDA.

Overall interventional techniques are escalating and are associated with complications; and complica- tions should never be minimized. Consequently, application of principles of accountable and value-based interventional pain management are crucial. Other developments include reducing over-regulation and applying appropriate regulations without shifting services from one sector to the other with evidence-based approaches.

\section{Conclusion}

Interventional techniques increased significantly in Medicare beneficiaries from 2000 to 2013. There was an increase of $156 \%$ in utilizing interventional pain management services per 100,000 fee-for-service Medicare beneficiaries, with an annual average increase of $7.5 \%$. The study also showed an exponential increase in facet joint interventions and sacroiliac joint blocks.

\section{Acknowledgments}

The authors wish to thank Tom Prigge, MA, and Laurie Swick, BS, for manuscript review, and Tonie M. Hatton and Diane E. Neihoff, transcriptionists, for their assistance in preparation of this manuscript. We would like to thank the editorial board of Pain Physician for review and criticism in improving the manuscript. 
Pain Physician: March/April 2015; 18:E115-E127

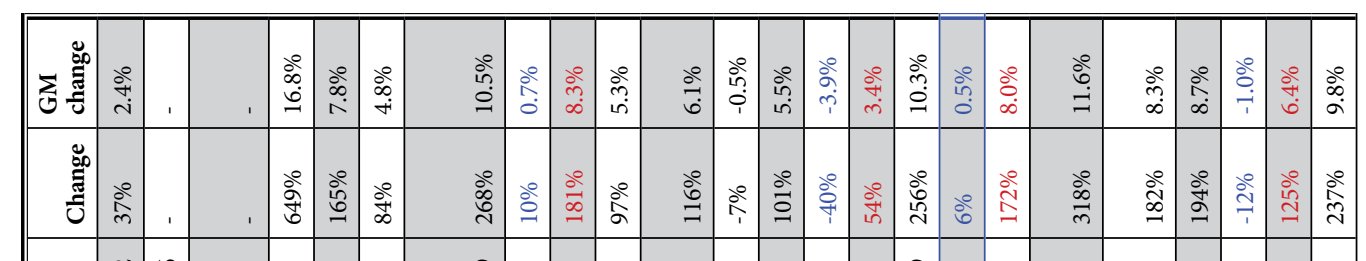

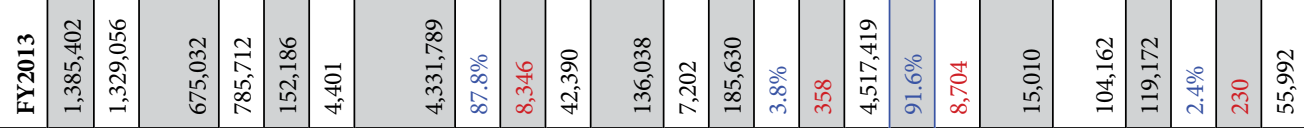

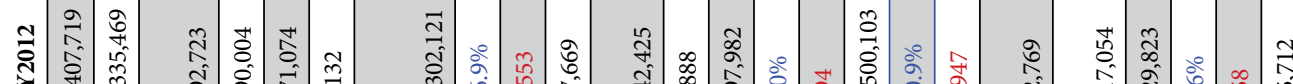

产

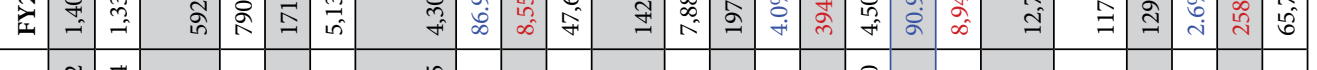

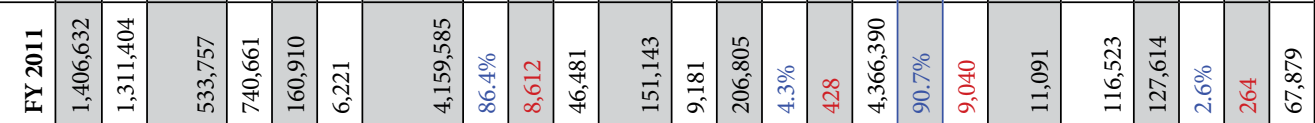

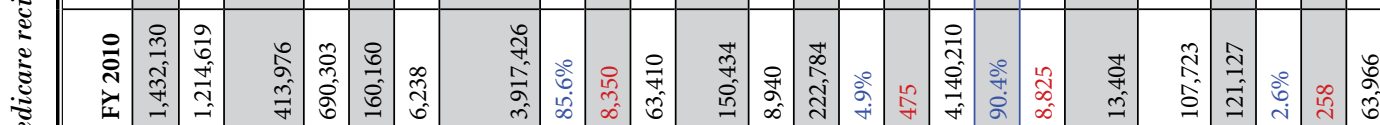

$\sum^{\frac{1}{5}}$

.

$\frac{\sqrt{1}}{\frac{1}{8}}$

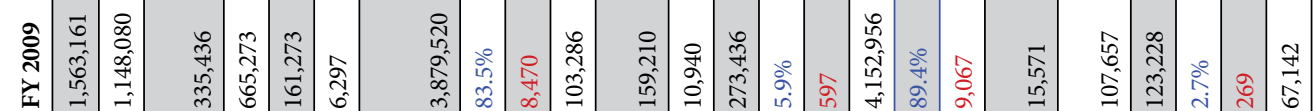

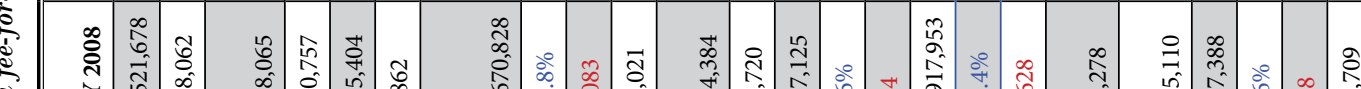

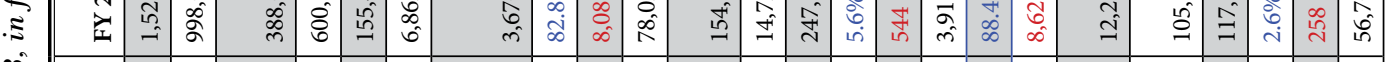

空

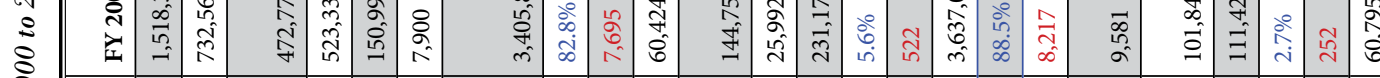

政

象

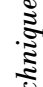

(2)

ฐ

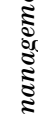

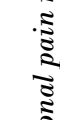

:

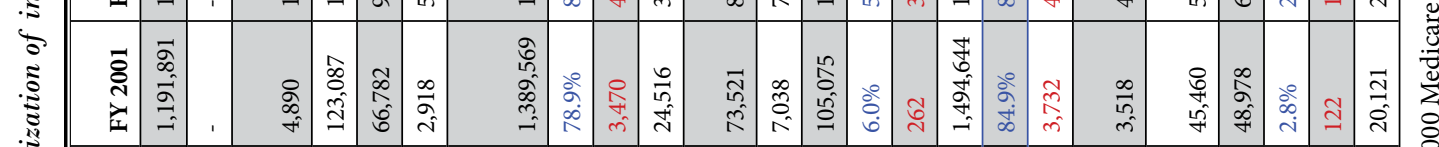

(1)

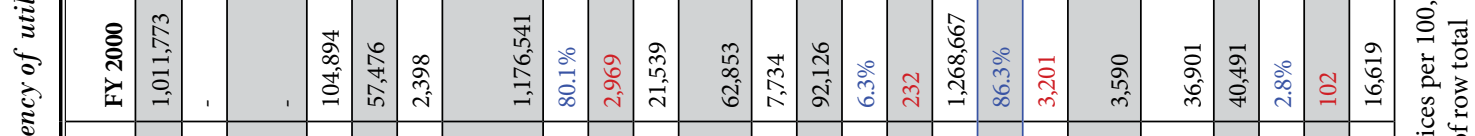

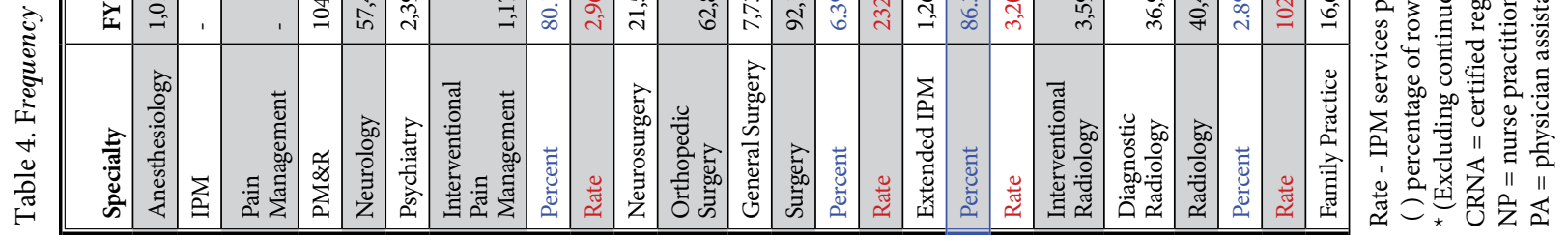




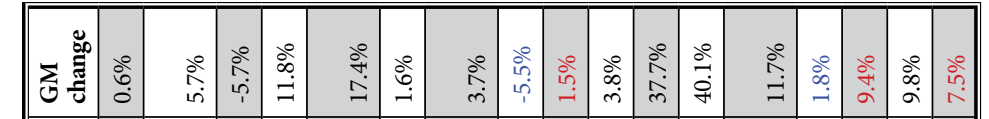

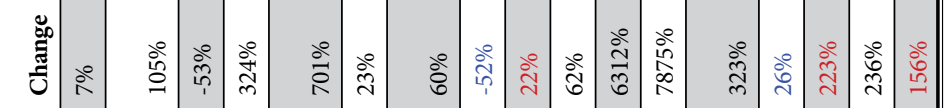

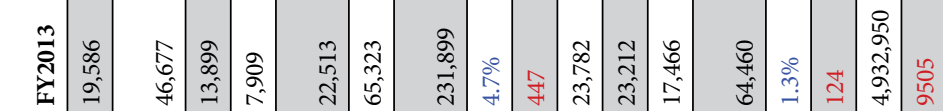

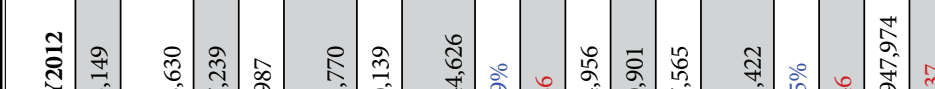

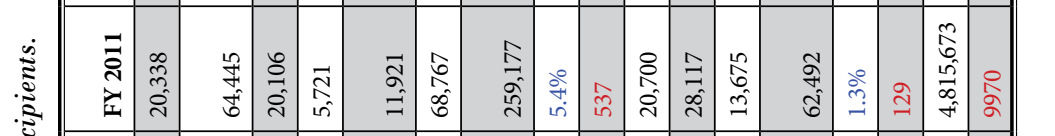

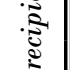

"ี

:

.

$\frac{a}{8}$

$\frac{8}{3}$

$$
\text { คุ }
$$

$$
\stackrel{8}{8}
$$

รั)

కี

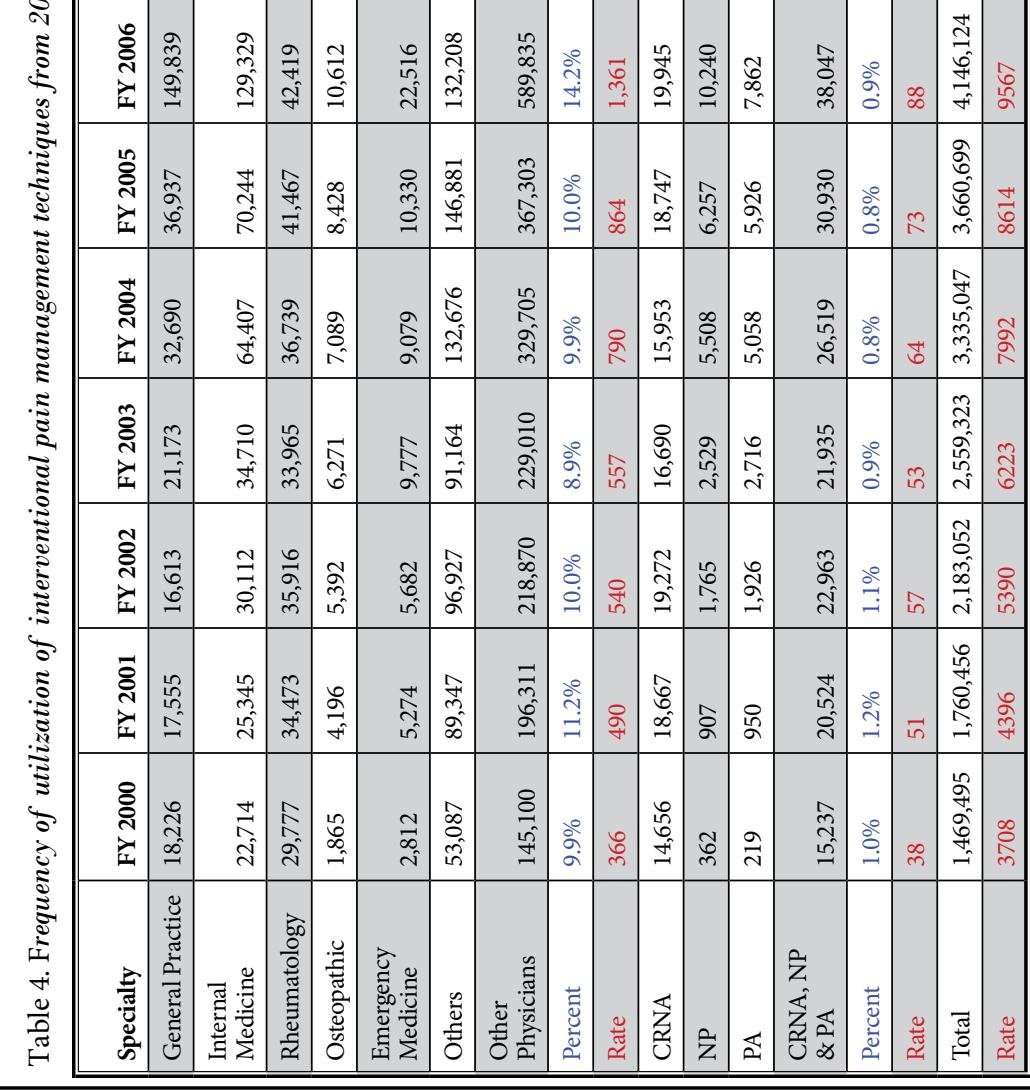




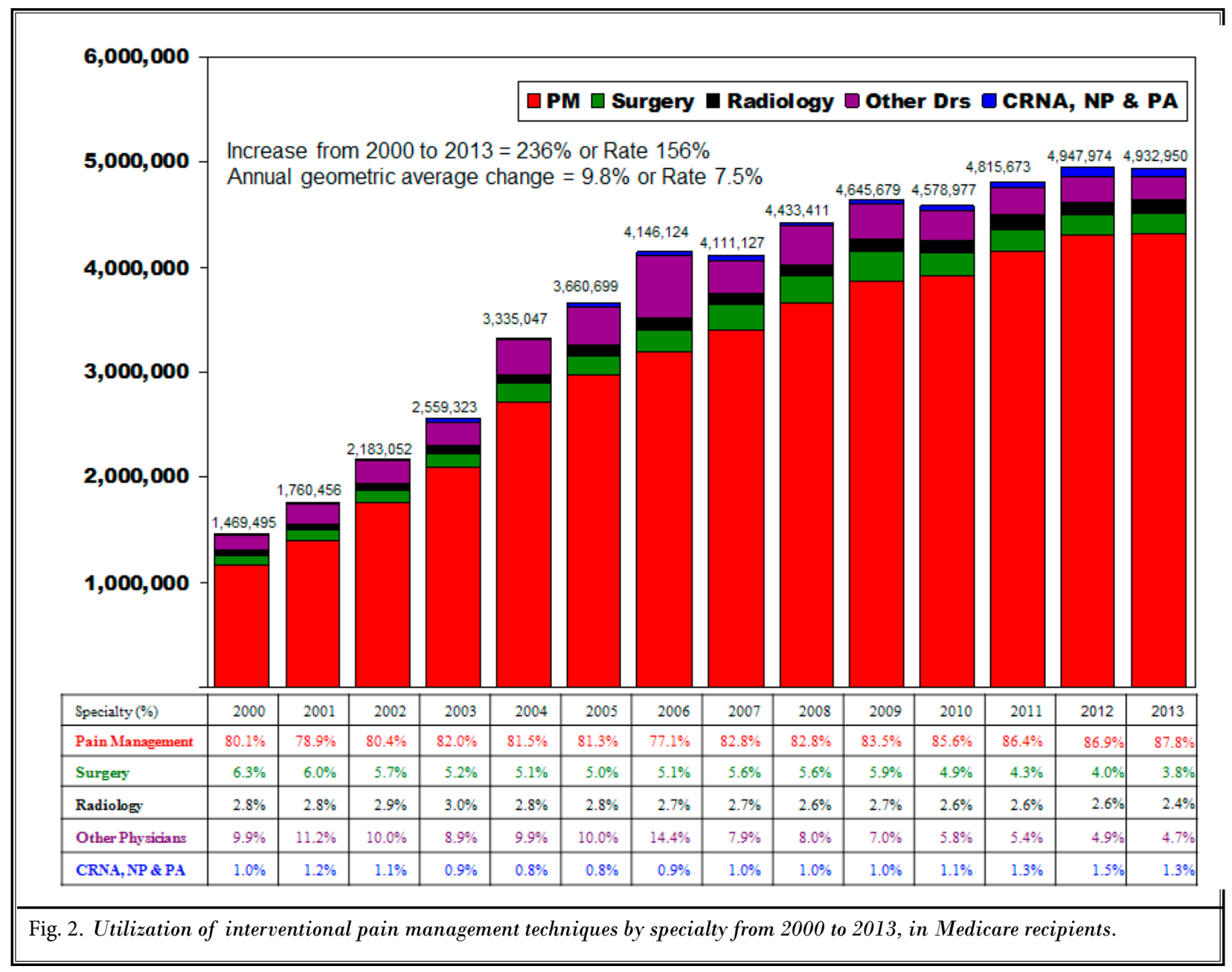

\section{Author affiliations:}

Dr. Manchikanti is Medical Director of the Pain Management Center of Paducah, Paducah, KY, and Clinical Professor, Anesthesiology and Perioperative Medicine, University of Louisville, Louisville, KY.

Vidyasagar Pampati is a Statistician at the Pain Management Center of Paducah, Paducah, KY.

Dr. Falco is Medical Director of Mid Atlantic Spine \& Pain Physicians, Newark, DE; Director, Pain Medicine Fellowship Program, Temple University Hospital, Phila- delphia, PA; and Adjunct Associate Professor, Department of PM\&R, Temple University Medical School, Philadelphia, PA.

Dr. Hirsch is Vice Chief of Interventional Care, Chief of Minimally Invasive Spine Surgery, Service Line Chief of Interventional Radiology, Director of Neurolnterventional Services and Neuroendovascular Program, Massachusetts General Hospital; and Associate Professor, Harvard Medical School, Boston, MA. 


\section{References}

1. Institute of Medicine (IOM). Relieving Pain in America: A Blueprint for Transforming Prevention, Care, Education, and Research. The National Academies Press, Washington, DC, June 29, 2011. www.iom.edu/ /media/Files/Report\%2oFiles/2011/Relieving-Pain-inAmerica-A-Blueprint-for-TransformingPrevention-Care-Education-Research/ Pain\%20Research\%202011\%20Report\%2oBrief.pdf

2. Gaskin DJ, Richard P. The economic costs of pain in the United States. J Pain 2012; 13:715-724.

3. US Burden of Disease Collaborators. The state of US health, 1999-2010: Burden of diseases, injuries, and risk factors. JAMA 2013; 310:591-608.

4. Buchbinder R, Blyth FM, March LM, Brooks P, Woolf AD, Hoy DG. Placing the global burden of low back pain in context. Best Pract Res Clin Rheumatol 2013; 27:575-589.

5. Freburger JK, Holmes GM, Agans RP, Jackman AM, Darter JD, Wallace AS, Castel LD, Kalsbeek WD, Carey TS. The rising prevalence of chronic low back pain. Arch Intern Med 2009; 169:251-258.

6. Sadosky AB, DiBonaventura M, Cappelleri JC, Ebata N, Fujii K. The association between lower back pain and health status, work productivity, and health care resource use in Japan. J Pain Res 2015; 8:119-130.

7. Hoy DG, Protani M, De R, Buchbinder R. The epidemiology of neck pain. Best Pract Res Clin Rheumatol 2010; 24:783-792.

8. Manchikanti L, Singh V, Falco FJE, Benyamin RM, Hirsch JA. Epidemiology of low back pain in adults. Neuromodulation 2014; 17:3-10.

9. Hoy D, March L, Brooks P, Blyth F, Woolf A, Bain C, Williams G, Smith E, Vos T, Barendregt J, Murray C, Burstein $\mathrm{R}$, Buchbinder R. The global burden of low back pain: estimates from the Global Burden of Disease 2010 study. Ann Rheum Dis 2014; 73:968-974.

10. U.S. Social Security Administration. Annual statistical report on the Social Security Disability Insurance Program, 2011. Release July 2012. www.ssa.gov/ policy/docs/statcomps/di_asr/2011/di_ asrı.pdf

11. Manchikanti L, Hirsch JA. Lessons learned in the abuse of pain relief medication: A focus on health care costs. Ex- pert Rev Neurother 2013; 13:527-544.

12. Manchikanti L, Abdi S, Atluri S, Benyamin RM, Boswell MV, Buenaventura RM, Bryce DA, Burks PA, Caraway DL, Calodney AK, Cash KA, Christo PJ, Cohen SP, Colson J, Conn A, Cordner HJ, Coubarous S, Datta S, Deer TR, Diwan SA, Falco FJE, Fellows B, Geffert SC, Grider JS, Gupta $S$, Hameed $H$, Hameed $M$, Hansen $H$, Helm II S, Janata JW, Justiz R, Kaye AD, Lee $M$, Manchikanti KN, McManus CD, Onyewu O, Parr AT, Patel VB, Racz GB, Sehgal N, Sharma M, Simopoulos TT, Singh V, Smith HS, Snook LT, Swicegood J, Vallejo R, Ward SP, Wargo BW, Zhu J, Hirsch JA. An update of comprehensive evidence-based guidelines for interventional techniques of chronic spinal pain: Part II: Guidance and recommendations. Pain Physician 2013; 16:S49-S283.

13. Martin BI, Deyo RA, Mirza SK, Turner JA, Comstock BA, Hollingworth W, Sullivan SD. Expenditures and health status among adults with back and neck problems. JAMA 2008; 299:656-664. Erratum in: JAMA 2008; 299:2630.

14. Martin BI, Turner JA, Mirza SK, Lee MJ, Comstock BA, Deyo RA. Trends in health care expenditures, utilization, and health status among US adults with spine problems, 1997-2006. Spine (Phila Pa 1976) 2009; 34:2077-2084.

15. Rajaee SS, Bae HW, Kanim LE, Delamarter RB. Spinal fusion in the United States: Analysis of trends from 1998 to 2008. Spine (Phila Pa 1976) 2012; 37:67-76.

16. Manchikanti L, Helm II S, Singh V, Hirsch JA. Accountable interventional pain management: A collaboration among practitioners, patients, payers, and government. Pain Physician 2013; 16:E635-E670.

17. Manchikanti L, Pampati V, Falco FJE, Hirsch JA. Growth of spinal interventional pain management techniques: Analysis of utilization trends and Medicare expenditures 2000 to 2008. Spine (Phila Pa 1976) 2013; 38:157-168.

18. Abbott ZI, Nair KV, Allen RR, Akuthota VR. Utilization characteristics of spinal interventions. Spine ] 2012; 1:35-43.

19. Manchikanti L, Falco FJE, Singh V, Pampati V, Parr AT, Benyamin RM, Fellows B, Hirsch JA. Utilization of interventional techniques in managing chronic pain in the Medicare population: Analysis of growth patterns from 2000 to 2011 . Pain Physician 2012; 15:E969-E982.
20. Leavitt SB. NSAID dangers may limit pain-relief options. Pain-Topics News/ Research UPDATES, March 14, 2010. http://updates.pain-topics.org/2010/03/ nsaid-dangers-may-limit-pain-relief. html

21. Moore A, Wiffen P, Kalso E. Antiepileptic drugs for neuropathic pain and fibromyalgia. JAMA 2014;312:182-183.

22. Dart RC, Surratt HL, Cicero TJ, Parrino MW, Severtson SG, Bucher-Bartelson B, Green JL. Trends in opioid analgesic abuse and mortality in the United States. N Engl ] Med 2015; 372:241-248.

23. Manchikanti L, Candido KD, Singh V, Gharibo CG, Boswell MV, Benyamin RM, Falco FJE, Grider JS, Diwan $S$, Hirsch JA. Epidural steroid warning controversy still dogging FDA. Pain Physician 2014; 17:E451-E474.

24. Manchikanti L, Falco FJE, Benyamin RM, Gharibo CG, Candido KD, Hirsch JA. Epidural steroid injections safety recommendations by the Multi-Society Pain Workgroup (MPW): More regulations without evidence or clarification. Pain Physician 2014; 17: $\mathrm{E}_{575}-\mathrm{E}_{5} 88$.

25. U.S. Food and Drug Administration. Drug Safety Communications. FDA Drug Safety Communication: FDA requires label changes to warn of rare but serious neurologic problems after epidural corticosteroid injections for pain www.fda.gov/downloads/Drugs/DrugSafety/UCM394286.pdf

26. Food and Drug Administration. Anesthetic and Analgesic Drug Products Advisory Committee Meeting. November 24,-25, 2014. Epidural steroid injections (ESI) and the risk of serious neurologic adverse reactions.

www.fda.gov/downloads/Ad visoryCom mittees/Com mit tees Meeting Materials/Drugs/ AnestheticAndAnalgesicDrugProductsAdvisoryCommittee/UCM422692.pdf

27. Express Scripts Lab. A Nation in Pain. Focusing on U.S. Opioid Trends for Treatment of short-term and longerterm pain. December 2014.

http://lab.express-scripts.com/publications/ /media/d48ef3ee579848e7bf3fi4af536d7548.ashx

28. Thielke SM, Turner JA, Shortreed SM, Saunders K, Leresche L, Campbell Cl, Weisner CC, Korff MV. Do patient-perceived pros and cons of opioids predict sustained higher-dose use? Clin J Pain 
2014;30:93-101.

29. Centers for Disease Control and Prevention. CDC grand rounds: Prescription drug overdoses - a U.S. epidemic. MMWR Morb Mortal Wkly Rep 2012; 61:10-13.

30. Atluri S, Sudarshan G, Manchikanti L. Assessment of the trends in medical use and misuse of opioid analgesics from 2004 to 2011. Pain Physician 2014; 17:E119-E128.

31. Bachhuber MA, Saloner B, Cunningham $\mathrm{CO}$, Barry CL. Medical cannabis laws and opioid analgesic overdose mortality in the United States, 1999-2010. JAMA Intern Med 2014; 174:1668-1673

32. Yokell MA, Delgado MK, Zaller ND, Wang NE, McGowan SK, Green TC. Presentation of prescription and nonprescription opioid overdoses to US emergency departments. JAMA Intern Med 2014; 174:2034-2037.

33. Pettit AC, Malani AN. Outbreak of fungal infections associated with contaminated methylprednisolone acetate: An update. Curr Infect Dis Rep 2015; 17:441.

34. Rathmell JP, Benzon HT, Dreyfuss P, Huntoon M, Wallace M, Baker R, Riew $\mathrm{KD}$, Rosenquist RW, Aprill C, Rost NS, Buvanendran A, Kreiner DS, Bogduk N, Fourney DR, Fraifeld E, Horn S, Stone J, Vorenkamp K, Lawler G, Summers J, Kloth D, O'Brien D Jr, Tutton S. Safeguards to prevent neurologic complications after epidural steroid injections: consensus opinions from a multidisciplinary working group and national organizations. Anesthesiology 2015 Feb 9. [Epub ahead of print]

35. US Department of Health and Human Services. Office of Inspector General (OIG). Medicare Payments for Facet Joint Injection Services (OEI-05-0700200). September 2008. www.oig.hhs. gov/oei/reports/oei-05-07-00200.pdf

36. US Department of Health and Human Services. Office of Inspector General (OIG). Inappropriate Medicare Payments for Transforaminal Epidural Injection Services (OEI-05-09-00030). August 2010. http:/oig.hhs.gov/oei/ reports/oei-05-09-00030.pdf

37. The National Uniform Claims Committee. Specialty Designation for Interventional Pain Management -o9.

38. Medicare Payment Advisory Commis sion Report to the Congress. Paying for Interventional Pain Services in Ambulatory Settings. December 2001.

39. US Department of Health and Human Services. Centers for Medicare and
Medicaid Services (CMS) Manual System. Pub. 100-08 Medicare Program Integrity. Inclusion of Interventional Pain Management Specialists on Carrier Advisory Committee (CAC) Membership. Change request 3721. March 4, 2005. www.cms.hhs.gov/transmittals/downloads/R106PI.pdf

40. Manchikanti L, Falco FJE, Benyamin RM, Helm II S, Singh V, Hirsch JA. Value-based interventional pain management: A review of Medicare national and local coverage determination policies. Pain Physician 2013; 16:E145-E180.

41. Manchikanti L, Hansen H, Benyamin RM, Falco FJE, Kaye AD, Hirsch JA. Declining value of work of interventional pain physicians. Pain Physician 2014; 17:Eı1-Eı9.

42. Public Law No: 111-148: H.R. 3590. Patient Protection and Affordable Care Act. March 23, 2010.

43. Manchikanti L, Hirsch JA. Regulatory burdens of the Affordable Care Act. Harvard -Health Policy Rev 2012; 13:9-12.

44. Macvicar J, King W, Landers MH, Bogduk N. The effectiveness of lumbar transforaminal injection of steroids: A comprehensive review with systematic analysis of the published data. Pain Med 2013; 14:14-28.

45. Manchikanti L, Benyamin RM, Falco FJ, Kaye AD, Hirsch JA. Do epidural injections provide short- and long-term relief for lumbar disc herniation? A systematic review. Clin Orthop Relat Res 2014 Feb 11. [Epub ahead of print].

46. Manchikanti L, Nampiaparampil DE, Candido KD, Bakshi S, Grider JS, Falco FJE, Sehgal N, Hirsch JA. Do cervical epidural injections provide long-term relief in neck and upper extremity pain? A systematic review. Pain Physician 2015; 18:39-6o.

47. Manchikanti L, Nampiaparampil DE, Manchikanti KN, Falco FJE, Singh V, Benyamin RM, Kaye AD, Sehgal N, Soin A, Simopoulos TT, Bakshi S, Gharibo CG, Gilligan CJ, Hirsch JA. Comparison of the efficacy of saline, local anesthetics, and steroids in epidural and facet joint injections for the management of spinal pain: A systematic review of randomized controlled trials. Surg Neurol Int 2015; in press.

48. Manchikanti L, Kaye AD, Manchikanti KN, Boswell MV, Pampati V, Hirsch JA. Efficacy of epidural injections in the treatment of lumbar central spinal stenosis: A systematic review. Anesth Pain Med 2015; in press.
49. Chou R, Hashimoto R, Friedly J, Fu R, Dana T, Elliott S, Sullivan S, Jarvik J. Pain Management Injection Therapies for Low Back Pain. Technology Assessment Report Prepared for Agency for Healthcare Research and Quality (AHRQ). Project ID: ESIBo813. October 29, 2014.

50. Pinto RZ, Maher CG, Ferreira ML, Hancock M, Oliveira VC, McLachlan AJ, Koes $\mathrm{B}$, Ferreira $\mathrm{PH}$. Epidural corticosteroid injections in the management of sciatica: A systematic review and meta-analysis. Ann Intern Med 2012; 157:865-877.

51. Chou R, Huffman L. Guideline for the Evaluation and Management of Low Back Pain: Evidence Review. American Pain Society, Glenview, IL, 2009. www.americanpainsociety.org/uploads/pdfs/LBPEvidRev.pdf

52. Staal JB, de Bie RA, de Vet HC, Hildebrandt J, Nelemans P. Injection therapy for subacute and chronic low back pain: An updated Cochrane review. Spine (Phila Pa 1976) 2009; 34:49-59.

53. Manchikanti L, Benyamin RM, Falco FJE, Caraway DL, Datta S, Hirsch JA. Guidelines warfare over interventional techniques: Is there a lack of discourse or straw man? Pain Physician 2012; 15:E1-E26.

54. Centers for Medicare and Medicaid Services.

www.cms.hhs.gov/home/medicare.Asp

55. Friedly J, Chan L, Deyo R. Increases in lumbosacral injections in the Medicare population: 1994 to 2001. Spine (Phila Pa 1976) 2007; 32:1754-176o.

56. Friedly J, Chan L, Deyo R. Geographic variation in epidural steroid injection use in Medicare patients. J Bone Joint Surg Am 2008; 90:1730-1737.

57. Medicare and Medicaid Statistical Supplement report that provides detailed statistical information on Medicare, Medicaid, and other Centers for Medicare \& Medicaid Services (CMS) programs.

www.cms.gov/Research-Statistics-Dataand-Systems/Statistics-Trends-and-Reports/MedicareMedicaidStatSuppMedicareMedicaidStatSupp/index.html

58. Manchikanti L, Pampati V, Singh V, Boswell MV, Smith HS, Hirsch JA. Explosive growth of facet joint interventions in the Medicare population in the United States: A comparative evaluation of 1997, 2002, and 2006 data. BMC Health Serv Res 2010; 10:84.

59. Manchikanti L, Pampati V, Boswell MV, Smith HS, Hirsch JA. Analysis of the growth of epidural injections and costs 
in the Medicare population: A comparative evaluation of 1997, 2002, and 2006 data. Pain Physician 2010; 13:199-212.

6o. Manchikanti L, Singh V, Pampati V, Smith HS, Hirsch JA. Analysis of growth of interventional techniques in managing chronic pain in Medicare population: A 10-year evaluation from 1997 to 2006. Pain Physician 2009; 12:9-34.

61. Manchikanti L, Boswell MV, Giordano J. Re: Friedly J, Chan L, Deyo R. Increases in lumbosacral injections in the Medicare population: 1994 to 2001. Spine (Phila PA 1976) 2007; 32:1754-1760. Spine (Phila Pa 1976) 2007; 32:3092.

62. Deyo RA, Mirza SK, Turner JA, Martin BI. Overtreating chronic back pain: Time to back off? J Am Board Fam Med 2009; 22:62-68.

63. Yoshihara $\mathrm{H}$, Yoneoka D. National trends in the surgical treatment for lumbar degenerative disc disease: United States, 2000 to 2009. Spine ] 2015; 15:265-271.

64. Deyo RA. Fusion surgery for lumbar degenerative disc disease: Still more questions than answers. Spine J 2015; 15 : 272-274.

65. Bekkering GE, Bala MM, Reid K, Kel- len E, Harker J, Riemsma R, Huygen FJ, Kleijnen J. Epidemiology of chronic pain and its treatment in The Netherlands. Neth J Med 2011; 69:141-153.

66. Reid KJ, Harker J, Bala MM, Truyers C, Kellen E, Bekkering GE, Kleijnen J. Epidemiology of chronic non-cancer pain in Europe: Narrative review of prevalence, pain treatments and pain impact. Curr Med Res Opin 2011; 27:449-462.

67. Langley PC. The prevalence, correlates and treatment of pain in the European Union. Curr Med Res Opin 2011; 27:463-480.

68. Tosato M, Lukas A, van der Roest HG, Danese P, Antocicco M, Finne-Soveri $\mathrm{H}$, Nikolaus T, Landi F, Bernabei R, Onder G. Association of pain with behavioral and psychiatric symptoms among nursing home residents with cognitive impairment: Results from the SHELTER study. Pain 2012; 153:305-310.

69. Bressler HB, Keyes WJ, Rochon PA, Badley $E$. The prevalence of low back pain in the elderly. A systemic review of the literature. Spine (Phila Pa 1976) 1999; 24:1813-1819.

70. Harker J, Reid KJ, Bekkering GE, Kel-
Ien $E$, Bala MM, Riemsma R, Worthy G, Misso K, Kleijnen J. Epidemiology of chronic pain in Denmark and Sweden. Pain Res Treat 2012; 2012:371248.

71. Reitsma M, Tranmer JE, Buchanan DM, VanDenKerkhof EG. The epidemiology of chronic pain in Canadian men and women between 1994 and 2007: Longitudinal results of the National Population Health Survey. Pain Res Manag 2012; 17:166-172.

72. Kurita GP, Sjøgren P, Juel K, Højsted J, Ekholm O. The burden of chronic pain: A cross-sectional survey focussing on diseases, immigration, and opioid use. Pain 2012; 153:2332-2338.

73. Leboeuf-Yde C, Fejer R, Nielsen J, Kyvik $\mathrm{KO}$, Hartvigsen J. Pain in the three spinal regions: The same disorder? Data from a population-based sample of 34,902 Danish adults. Chiropr Man Therap 2012; 20:11.

74. Manchikanti L, Atluri S, Candido KD, Boswell MV, Simopoulos TT, Grider JS, Falco FJE, Hirsch JA. Zohydro'M approval by Food and Drug Administration: Controversial or frightening? Pain Physician 2014; 17:E437-E450. 
